\title{
Associations of types of dairy consumption with adiposity: cross-sectional findings from over 12000 adults in the Fenland Study, UK
}

\author{
Eirini Trichia ${ }^{1 *}$, Fumiaki Imamura ${ }^{1}$, Søren Brage ${ }^{1}$, Emanuella De Lucia Rolfe ${ }^{1}$, Simon J. Griffin ${ }^{1,2}$, \\ Nicholas J. Wareham ${ }^{1}$ and Nita G. Forouhi ${ }^{1 *}$ \\ ${ }^{1}$ MRC Epidemiology Unit, Institute of Metabolic Science, University of Cambridge School of Clinical Medicine, Cambridge, UK \\ ${ }^{2}$ Department of Public Health and Primary Care, Institute of Public Health, University of Cambridge School of Clinical Medicine, \\ Cambridge, UK
}

(Submitted 17 December 2018 - Final revision received 12 July 2019 - Accepted 18 July 2019)

\section{Abstract}

Evidence from randomised controlled trials supports beneficial effects of total dairy products on body weight, fat and lean mass, but evidence on associations of dairy types with distributions of body fat and lean mass is limited. We aimed to investigate associations of total and different types of dairy products with markers of adiposity, and body fat and lean mass distribution. We evaluated cross-sectional data from 12065 adults aged 30-65 years recruited to the Fenland Study between 2005 and 2015 in Cambridgeshire, UK. Diet was assessed with an FFQ. We estimated regression coefficients (or percentage differences) and their $95 \% \mathrm{CI}$ using multiple linear regression models. The medians of milk, yogurt and cheese consumption were 293 (interquartile range (IQR) 146-439), 35.3 (IQR 8.8-71.8) and 14.6 (IQR 4.8-26.9) g/d, respectively. Low-fat dairy consumption was inversely associated with visceral:subcutaneous fat ratio estimated with dual-energy X-ray absorptiometry $(-2 \cdot 58 \%$ (95\% CI $-3.91,-1.23 \%$ ) per serving/d). Habitual consumption per serving/d (200 g) of milk was associated with 0.33 (95\% CI 0.19 , 0.46 ) $\mathrm{kg}$ higher lean mass. Other associations were not significant after false discovery correction. Our findings suggest that the influence of milk consumption on lean mass and of low-fat dairy consumption on fat mass distribution may be potential pathways for the link between dairy consumption and metabolic risk. Our cross-sectional findings warrant further research in prospective and experimental studies in diverse populations.

Key words: Dairy products: Milk: Yogurt: Cheese: Butter: Adiposity

Diet is acknowledged to be a leading behavioural risk factor for cardiometabolic diseases ${ }^{(1)}$. Among dietary factors, dairy products are of particular interest due to their inverse association with cardiometabolic health ${ }^{(2)}$, thought to be related to their high nutrient density and mineral content, but also due to the controversy arising from their blood cholesterol raising saturated fat content $^{(3)}$. Several meta-analyses of prospective cohort studies on the associations of dairy products with $\mathrm{CVD}^{(4,5)}$ and type 2 diabetes $^{(6-8)}$ have suggested that the associations vary by dairy type. There is a general concordance of observational results suggesting an inverse association between yogurt consumption and type 2 diabetes $^{(6-8)}$. In contrast, evidence for associations between other dairy types and type 2 diabetes ${ }^{(7,9)}$ or $\mathrm{CVD}^{(4)}$ is inconsistent with either null or inverse associations reported for the consumption of cheese and low-fat milk ${ }^{(4,7,9)}$.

Adiposity is one of the most well-described potential pathways which might link total dairy consumption to cardiometabolic disease pathogenesis. Evidence from randomised clinical trials shows that total dairy products decrease body weight ${ }^{(10)}$ and body fat mass ${ }^{(10)}$, and increase body lean mass ${ }^{(11)}$ under energy restriction. However, evidence on associations between types of dairy products and markers of adiposity, especially markers of fat and lean mass distribution, is sparse. Visceral adipose tissue (VAT) and subcutaneous adipose tissue (SCAT) are compartments of abdominal fat. While abdominal fat is associated with cardiometabolic diseases ${ }^{(12)}$, VAT has been more strongly associated with cardiometabolic risk than SCAT ${ }^{(13)}$, which has been suggested to be potentially protective ${ }^{(14)}$. Specifically the ratio of VAT to SCAT was reported to be strongly associated with cardiometabolic risk independent of BMI and VAT ${ }^{(15)}$. Due to the higher cost of the methods to estimate abdominal fat distribution, for example, dual-energy X-ray absorptiometry (DEXA), most large-scale population studies examine waist circumference as a proxy for abdominal fat, which cannot fully capture the distribution between VAT and SCAT. Only

Abbreviations: DEXA, dual-energy X-ray absorptiometry; SCAT, subcutaneous adipose tissue; VAT, visceral adipose tissue.

* Corresponding authors: Dr E. Trichia, fax +44 1223 330316, email Eirini.Trichia@mrc-epid.cam.ac.uk; Professor N. G. Forouhi, fax +44 1223 330316, email Nita.Forouhi@mrc-epid.cam.ac.uk 


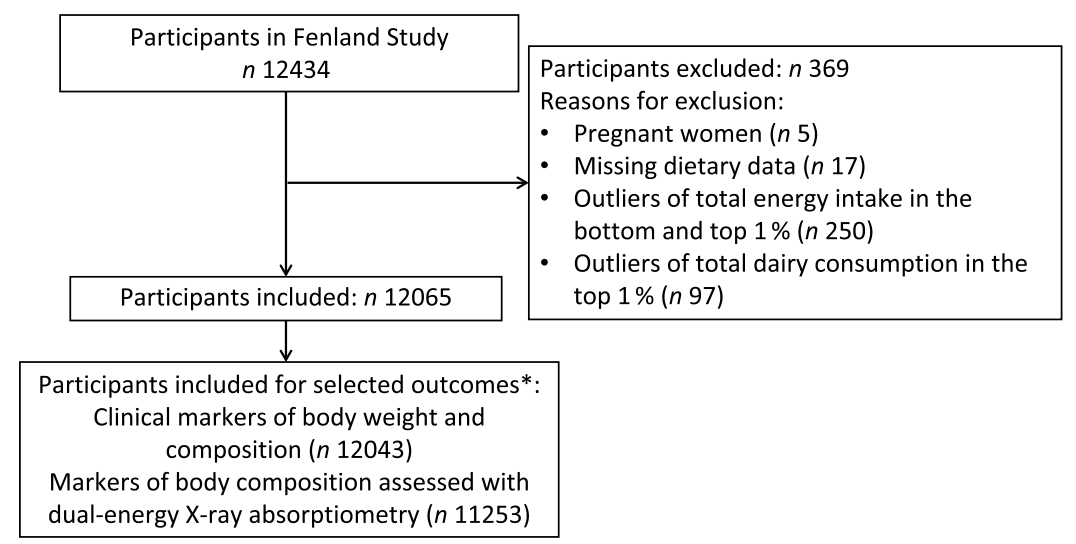

Fig. 1. Participant selection for analyses of associations of dairy consumption with cardiometabolic markers in over 12000 adults of the Fenland Study, UK. *A minimal number of participants for each sub-group of the outcomes is presented. Numbers slightly varied depending on missing information of each outcome variable.

a few studies have investigated associations between dairy products (total ${ }^{(16)}$ and low-fat ${ }^{(17)}$ ) and VAT, but evidence is lacking on associations of dairy consumption with SCAT and VAT:SCAT.

We aimed to investigate the associations of total and different types of dairy products with body fat and lean mass and their distribution in a large population-based study in the UK.

\section{Methods}

\section{Study design and population}

The Fenland Study is a cohort study with baseline measurements conducted between 2005 and 2015 ( $n 12434$ with $27 \%$ response rate). Eligible participants were born between 1950 and 1975 and were invited via their general practice to attend the clinical sites at Ely, Wisbech, or Cambridge, UK, where the clinical and dietary assessments were conducted. Exclusion criteria, assessed by general practitioners, were known history of diabetes, psychotic or terminal illness, inability to walk unaided and pregnancy or lactation. The study was approved by the Cambridge Local Ethics Committee. All participants gave written informed consent.

For the present analysis, we evaluated baseline data from 12065 participants after exclusion of pregnant women ( $n$ 5), participants with missing dietary data $(n 17)$, those in the bottom and top $1 \%$ of total energy intake ( $n$ 250) and those in the top $1 \%$ of total dairy consumption ( $n$ 97). Participants with missing outcome data were also excluded ( $n 1$ to $n$ 812, depending on the outcome). A study flow chart of participant inclusion is presented in Fig. 1.

\section{Dietary assessment}

Participants' diet over the previous year was assessed with a 130-item semi-quantitative FFQ ${ }^{(18)}$. The dietary data were processed using FETA software ${ }^{(19)}$. Dairy products were assessed in servings/d and were categorised as we previously described ${ }^{(20)}$ (Table 1). Dairy servings were defined as one average glass (200 g) for milk, $125 \mathrm{~g}$ pot for yogurt, a medium serving of $40 \mathrm{~g}$ for cheese, one tablespoon (15 g) for single cream, one tablespoon (30 g) for double cream, one teaspoon (10 g) for butter and one average scoop/tub (60 g) for ice cream.

\section{Adiposity measures}

These measurements were conducted at each clinical site according to standardised procedures. BMI was calculated as weight divided by height squared $\left(\mathrm{kg} / \mathrm{m}^{2}\right)$. Waist and hip circumferences were averaged from two repeated measures with a nonstretch tape. Total body fat and its distribution (peripheral fat, VAT, SCAT) and lean mass and its distribution (appendicular lean mass) were estimated with a DEXA scan (Lunar Prodigy Advanced fan beam scanner, GE Healthcare; GE encore software, version 14.10.022 to 16, GE Medical Systems ${ }^{(21)}$ ). Specifically, the DEXA system demarcated the boundaries of the android region based on an established protocol. VAT was estimated as part of the android region from an inbuilt algorithm of the DEXA software. SCAT was calculated after subtracting VAT from the android fat mass. These DEXA estimates of abdominal VAT and SCAT have been validated against amounts of VAT and SCAT determined by computed tomography ${ }^{(22)}$ and $\mathrm{MRI}^{(23)}$, the reference methods for adipose tissue quantification.

\section{Assessment of sociodemographic and lifestyle factors}

A general questionnaire was administered for information on ethnicity, occupation, education, income, marital status, smoking and medication use. Physical activity was objectively measured over $7 \mathrm{~d}$ using a combined heart rate and movement sensor (Actiheart, CamNtech) ${ }^{(24)}$ and individually calibrated with a treadmill test to derive physical activity energy expenditure $(\mathrm{kJ} / \mathrm{kg} \text { per } \mathrm{d})^{(25)}$. This method was previously validated with doubly labelled water.

\section{Statistical analyses}

As primary exposures, we considered milk, yogurt and cheese. As primary outcome, we defined the ratio of VAT to SCAT (VAT: SCAT). Other outcomes included total and peripheral body fat mass, total and appendicular body lean mass and proxies of abdominal fat, that is, waist circumference and the waist:hip ratio. We presented results for VAT:SCAT as percentage change after 


\section{British Journal of Nutrition}

Table 2. Descriptive characteristics of sociodemographic, behavioural and clinical factors* for the bottom (non-consumers) and top categories of milk, yogurt and cheese consumption (g/d), as well as in the total sample of 12065 adults of the Fenland Study, UK

(Medians and interquartile ranges (IQR); column percentages)

\begin{tabular}{|c|c|c|c|c|c|c|c|c|c|c|c|c|c|c|}
\hline \multirow{4}{*}{$\begin{array}{l}\text { Median (IQR) } \\
\text { consumption }(\mathrm{g} / \mathrm{d}) \ldots\end{array}$} & & & \multicolumn{4}{|c|}{ Milk† (g/d) } & \multicolumn{4}{|c|}{ Yogurt† (g/d) } & \multicolumn{4}{|c|}{ Cheese† (g/d) } \\
\hline & & & \multicolumn{4}{|c|}{$293(146-439)$} & \multicolumn{4}{|c|}{$35.3(8 \cdot 8-71 \cdot 8)$} & \multicolumn{4}{|c|}{$14.6(4 \cdot 8-26 \cdot 9)$} \\
\hline & \multicolumn{2}{|c|}{ Overall } & \multicolumn{2}{|c|}{ Bottom: $0 \mathrm{~g} / \mathrm{d}$} & \multicolumn{2}{|c|}{ Top: $585-732 \mathrm{~g} / \mathrm{d}$} & \multicolumn{2}{|c|}{ Bottom: $0 \mathrm{~g} / \mathrm{d}$} & \multicolumn{2}{|c|}{ Top: $99.5-1134 \mathrm{~g} / \mathrm{d}$} & \multicolumn{2}{|c|}{ Bottom: $0 \mathrm{~g} / \mathrm{d}$} & \multicolumn{2}{|c|}{ Top: $26.8-284 \mathrm{~g} / \mathrm{d}$} \\
\hline & Median & IQR & Median & IQR & Median & IQR & Median & IQR & Median & IQR & Median & IQR & Median & IQR \\
\hline $\begin{array}{l}\text { Participants }(n) \\
\text { Sociodicmographic factors }\end{array}$ & \multicolumn{2}{|c|}{12065} & \multicolumn{2}{|r|}{921} & \multicolumn{2}{|c|}{1490} & \multicolumn{2}{|c|}{2787} & \multicolumn{2}{|c|}{3014} & \multicolumn{2}{|r|}{779} & \multicolumn{2}{|r|}{3028} \\
\hline Age (years) & 48.8 & $42 \cdot 7-54 \cdot 7$ & $49 \cdot 6$ & $42 \cdot 8-55 \cdot 1$ & $48 \cdot 0$ & $42 \cdot 2-54 \cdot 0$ & 48.6 & $42 \cdot 4-54.6$ & $50 \cdot 0$ & $43 \cdot 7-55 \cdot 2$ & $48 \cdot 8$ & $42 \cdot 4-55 \cdot 2$ & $48 \cdot 1$ & $41 \cdot 9-54 \cdot 3$ \\
\hline Sex (ref. men): women & \multicolumn{2}{|r|}{53.8} & \multicolumn{2}{|r|}{63.7} & \multicolumn{2}{|r|}{38.7} & & 39.5 & & $65 \cdot 7$ & & $49 \cdot 0$ & & $60 \cdot 2$ \\
\hline Ethnicity $\ddagger$ (ref. White): non-White & & 2.9 & & 4.9 & & 1.4 & & 3.3 & & 1.8 & & 7.9 & & 1.6 \\
\hline Educational levelł (ref. low) & & & & & & & & & & & & & & \\
\hline Medium & & $46 \cdot 2$ & & 41.8 & & 48.0 & & $49 \cdot 0$ & & $46 \cdot 7$ & & $48 \cdot 1$ & & 41.9 \\
\hline High & & 33.9 & & $40 \cdot 7$ & & 27.5 & & $25 \cdot 0$ & & 33.5 & & 23.9 & & $42 \cdot 1$ \\
\hline $\begin{array}{l}\text { Age completing education (years) } \ddagger \\
\text { Socio-economic status (based on } \\
\text { occupation)§ (ref. low) }\end{array}$ & $18 \cdot 0$ & $16 \cdot 0-21 \cdot 0$ & $18 \cdot 0$ & $16 \cdot 0-22 \cdot 0$ & $17 \cdot 0$ & $16 \cdot 0-1 \cdot 0$ & $17 \cdot 0$ & $16 \cdot 0-20 \cdot 0$ & $18 \cdot 0$ & $16 \cdot 0-21 \cdot 0$ & $16 \cdot 5$ & $16 \cdot 0-19 \cdot 0$ & $18 \cdot 0$ & $16 \cdot 0-22 \cdot 0$ \\
\hline Medium & & $19 \cdot 8$ & & $19 \cdot 7$ & & $14 \cdot 6$ & & $16 \cdot 4$ & & $23 \cdot 1$ & & $21 \cdot 2$ & & 19.8 \\
\hline High & & $53 \cdot 3$ & & $58 \cdot 3$ & & 47.6 & & $46 \cdot 1$ & & 52.5 & & $42 \cdot 7$ & & 58.4 \\
\hline Income $\ddagger$ (ref. $<£ 20000$ ) & & & & & & & & & & & & & & \\
\hline$£ 20000-40000$ & & 35.4 & & $32 \cdot 6$ & & 37.5 & & 37.8 & & 37.3 & & 38.5 & & 34.4 \\
\hline$>£ 40000$ & & $50 \cdot 8$ & & $52 \cdot 0$ & & $47 \cdot 3$ & & $45 \cdot 1$ & & $49 \cdot 6$ & & $41 \cdot 9$ & & $52 \cdot 1$ \\
\hline Marital status $\|$ (ref. single) & & & & & & & & & & & & & & \\
\hline Married & & 81.4 & & $75 \cdot 1$ & & $80 \cdot 5$ & & 79.9 & & $80 \cdot 8$ & & $77 \cdot 1$ & & $80 \cdot 2$ \\
\hline Widowed/separated & & 9.5 & & $10 \cdot 3$ & & 9.9 & & $9 \cdot 3$ & & $10 \cdot 3$ & & 9.6 & & 9.8 \\
\hline Lifestyle factors & & & & & & & & & & & & & & \\
\hline Smoking status $\ddagger$ (ref. never) & & & & & & & & & & & & & & \\
\hline Former & & 33.3 & & $35 \cdot 6$ & & 31.8 & & 32.0 & & 34.0 & & $27 \cdot 6$ & & 34.3 \\
\hline Current & & $12 \cdot 3$ & & $13 \cdot 3$ & & $17 \cdot 8$ & & $20 \cdot 1$ & & $7 \cdot 6$ & & 13.5 & & $11 \cdot 1$ \\
\hline Smoking (pack-years)§ & 0.0 & $0.0-2376$ & 0.0 & $0.0-2696$ & 0.0 & $0.0-3701$ & 0.0 & $0.0-4362$ & 0.0 & $0.0-1414$ & 0.0 & $0.0-2725$ & 0.0 & $0.0-2192$ \\
\hline $\begin{array}{l}\text { Physical activity energy expenditure } \\
(\mathrm{kJ} / \mathrm{kg} \text { per d) } \ddagger \emptyset\end{array}$ & $50 \cdot 7$ & $37 \cdot 6-66 \cdot 5$ & $49 \cdot 9$ & $38 \cdot 1-63.5$ & 55.4 & $40 \cdot 8-72 \cdot 4$ & $51 \cdot 2$ & $37 \cdot 7-67 \cdot 8$ & $50 \cdot 5$ & $37 \cdot 0-65 \cdot 4$ & $49 \cdot 4$ & $35 \cdot 7-65 \cdot 7$ & $52 \cdot 0$ & $38 \cdot 8-67 \cdot 6$ \\
\hline Energy intake $(\mathrm{kJ} / \mathrm{d})$ & 1851 & $1524-2265$ & 1600 & $1312-2030$ & 2140 & $1733-2613$ & 1768 & $1433-2180$ & 1932 & $1603-2345$ & 1681 & 1360-2081 & 2067 & $1691-2507$ \\
\hline BMI $\left(\mathrm{kg} / \mathrm{m}^{2}\right)$ & $26 \cdot 2$ & $23 \cdot 6-29 \cdot 4$ & $25 \cdot 8$ & $23 \cdot 0-29 \cdot 1$ & $26 \cdot 7$ & $24 \cdot 2-29 \cdot 9$ & $26 \cdot 6$ & $23 \cdot 8-29 \cdot 6$ & $26 \cdot 1$ & $23 \cdot 5-29 \cdot 3$ & $26 \cdot 5$ & $23 \cdot 8-29 \cdot 6$ & $25 \cdot 8$ & $23 \cdot 2-29 \cdot 1$ \\
\hline Medications/supplements & & & & & & & & & & & & & & \\
\hline Lipid-lowering medication $\ddagger$ (ref. no): yes & & 4.0 & & 4.0 & & 3.0 & & 4.7 & & 4.0 & & 4.4 & & $3 \cdot 3$ \\
\hline Antihypertensive medication $\ddagger$ (ref. no): yes & & 7.4 & & 7.4 & & $6 \cdot 3$ & & $8 \cdot 2$ & & $7 \cdot 1$ & & $7 \cdot 8$ & & $6 \cdot 9$ \\
\hline $\begin{array}{l}\text { Hormonal therapy } \neq \text { (ref. no for } \\
\text { women/men): yes }\end{array}$ & & $2 \cdot 8$ & & 3.4 & & 1.9 & & $2 \cdot 0$ & & 3.5 & & $2 \cdot 7$ & & $3 \cdot 2$ \\
\hline Dietary supplementsł (ref. no): yes & & $41 \cdot 2$ & & 49.5 & & $40 \cdot 5$ & & 33.5 & & $47 \cdot 7$ & & 39.5 & & 44.5 \\
\hline
\end{tabular}

ref., Reference.

Continuous variables are presented as medians and IQR and categorical variables are presented as column percentages.

Five categories. Milk: non-consumers, 146, 293, 439, 585 or $732 \mathrm{~g} / \mathrm{d}$ (categories presented: non-consumers and 585-732 g/d). Yogurt: non-consumers and quartiles within consumers (1st quartile: 8.8-8.8 g/d, 2nd quartile: 17.6-35.3 g/d, 3rd quartile: $54.2-71.8 \mathrm{~g} / \mathrm{d}$, 4th quartile: $99.5-1134 \mathrm{~g} / \mathrm{d}$; categories presented: non-consumers and 4th quartile within consumers). Cheese: non-consumers and quartiles within consumers (1st quartile: $2.4-4.8 \mathrm{~g} / \mathrm{d}, 2 \mathrm{2nd}$ quartile: 6.6-13.2 g/d, 3rd

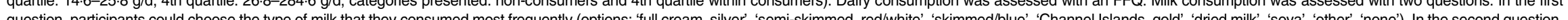
qurticipant pout, low Perc, low tat

(

Percentage of missing values $15-25 \%$ with a total of $28.8 \%$ of missing values when accounting for non-overlapping missing values across all the variables.

I Physical activity was objectively measured with a combined heart rate and movement sensor. 


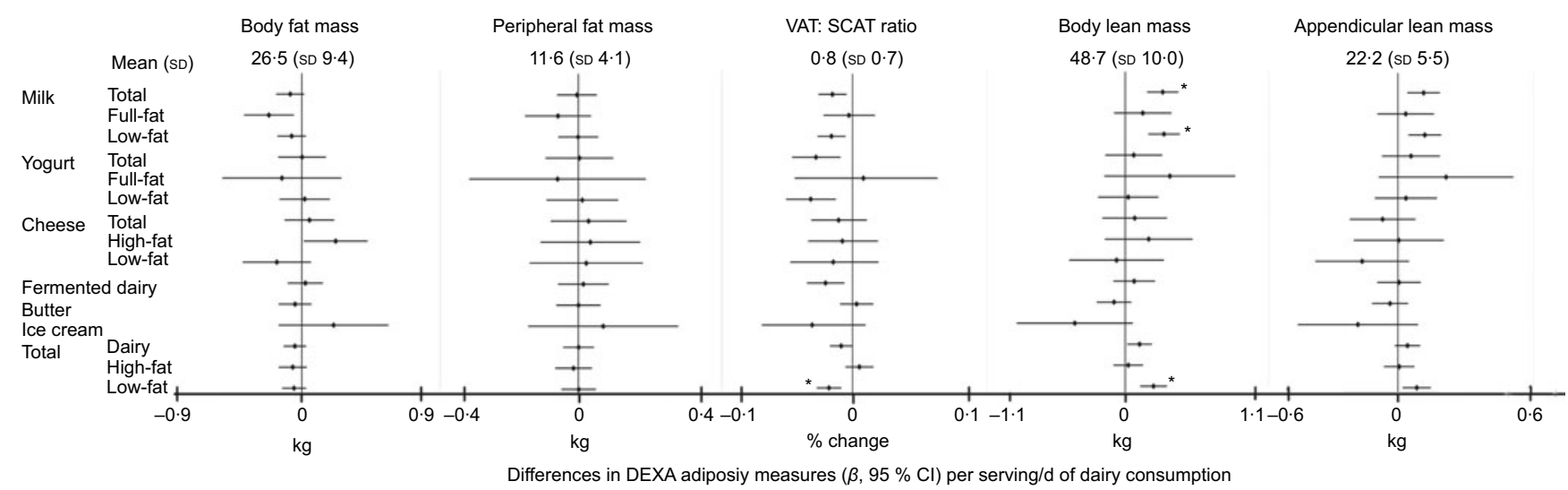

Fig. 2. Associations of types of dairy consumption (servings/d) with markers of body composition estimated with dual-energy X-ray absorptiometry (DEXA) among over 12000 adults in the Fenland Study, UK. Forest plots represent regression coefficients with their $95 \% \mathrm{Cl}$ adjusted for age (years), sex, test site (Cambridge, Ely, Wisbech), ethnicity (White, non-White), total energy intake ( $\mathrm{kJ} / \mathrm{d})$, other dairy types, educational level (low, medium, high), age when full-time education finished (years), socio-economic status based on occupation (low, medium, high), income ( $<£ 20000, £ 20000-40000,>£ 40000$ ), marital status (single, married, widowed/separated), smoking status (never, former, current smoker), pack-years of smoking, energy expenditure due to physical activity (kJ/kg per d), lipid-lowering medication (yes/no), antihypertensive medication (yes/no), hormone-replacement therapy (yes/no in women), intakes ( $\mathrm{g} / \mathrm{d}$ ) of fruit, vegetables, potatoes, legumes, processed cereals, wholegrain cereals, poultry and eggs, red meat, processed meat, fish, sauces, margarine, nuts, sweet snacks, sugar-sweetened beverages, artificially sweetened beverages, fruit juice, regular coffee, decaffeinated coffee, tea and alcoholic beverages, plasma vitamin C levels ( $\mu \mathrm{mol} / \mathrm{l})$, dietary supplement use (yes $/ \mathrm{no})$ and BMI $\left(\mathrm{kg} / \mathrm{m}^{2}\right)$. * Statistically significant associations after false discovery rate corrections. See categorisation of dairy types in Table 1. VAT, visceral adipose tissue; SCAT, subcutaneous adipose tissue.

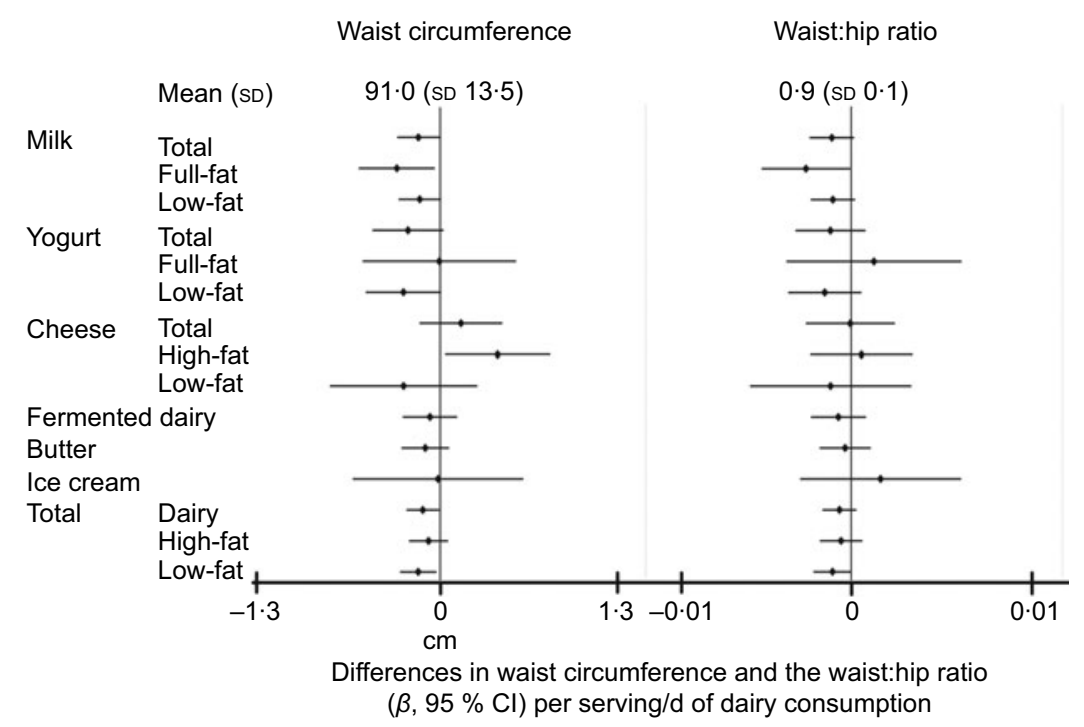

Fig. 3. Associations of types of dairy consumption (servings/d) with waist circumference and the waist:hip ratio among over 12000 adults in the Fenland Study, UK. Forest plots represent regression coefficients with their $95 \% \mathrm{Cl}$ adjusted for age (years), sex, test-site (Cambridge, Ely, Wisbech), ethnicity (White, non-White), total energy intake (kJ/d), other dairy types, educational level (low, medium, high), age when full-time education finished (years), socio-economic status based on occupation (low, medium, high), income ( $<£ 20000$, £20 000-40 000, >£40 000), marital status (single, married, widowed/separated), smoking status (never, former, current smoker), pack-years of smoking, energy expenditure due to physical activity ( $\mathrm{kJ} / \mathrm{kg}$ per d), lipid-lowering medication (yes/no), antihypertensive medication (yes/no), hormone-replacement therapy (yes/no in women), intakes $(\mathrm{g} / \mathrm{d})$ of fruit, vegetables, potatoes, legumes, processed cereals, whole-grain cereals, poultry and eggs, red meat, processed meat, fish, sauces, margarine, nuts, sweet snacks, sugar-sweetened beverages, artificially sweetened beverages, fruit juice, regular coffee, decaffeinated coffee, tea and alcoholic beverages, plasma vitamin C levels $(\mu \mathrm{mol} / \mathrm{l})$, dietary supplement use (yes $/ \mathrm{no})$ and BMI $\left(\mathrm{kg} / \mathrm{m}^{2}\right)$. See categorisation of dairy types in Table 1.

(Fig. 2). Low-fat milk showed similar associations (Fig. 2). The association was partly attenuated when adjusted for height, but it was still significant $(0 \cdot 18$ (95\% CI $0 \cdot 10,0 \cdot 27) \mathrm{kg} /$ serving of milk per d). Effect modification by BMI was suggested for the association between high-fat dairy products and appendicular lean mass ( $P$-interaction $=0 \cdot 0001)$. Among adults with nonoverweight BMI $\left(<25 \mathrm{~kg} / \mathrm{m}^{2}\right)$, appendicular lean mass was lower by $0 \cdot 11(95 \% \mathrm{CI}-0 \cdot 22,-0 \cdot 001) \mathrm{kg}$ per one serving of high-fat dairy product, but associations were not observed among overweight $(0 \cdot 05(95 \% \mathrm{CI}-0 \cdot 07,0 \cdot 18) \mathrm{kg})$ or obese adults (0.02 (95\% CI -0.16, 0.20) kg).

Other dairy types were not significantly associated with BMI, nor any dairy products with any other anthropometric measure (Figs. 2 and 3, Supplementary Fig. S1). 


\section{Other analyses}

Results were not altered when complete-case analyses were performed, and findings were similar when adjusting for the ratio of total energy intake to BMR rather than adjusting for BMI or after further adjustment for dairy nutrients (Supplementary Table S3). There was no indication of a non-linear association from analyses with restricted cubic splines or categorical exposures after correction for multiple testing.

\section{Discussion}

Our study reports two main findings. The first was that habitual daily consumption of one serving of low-fat dairy products was associated with a $3 \%$ lower ratio of VAT to SCAT as a marker of fat mass distribution, a measure which is associated with diabetes risk independently of total fat mass ${ }^{(15)}$. Second, habitual daily consumption of one serving of milk was associated with a 0.33 kg higher body lean mass.

There are no previous studies on the association between dairy consumption and VAT:SCAT. A randomised controlled trial showed a reduction in VAT among those consuming six to seven servings of dairy products per d compared with those consuming less than four servings/ $\mathrm{d}^{(16)}$, and a cross-sectional study of twins reported an inverse association between low-fat fermented dairy products and $\mathrm{VAT}^{(17)}$, but we have not identified any studies of the association between dairy consumption and SCAT. Although total dairy consumption has been consistently associated with a lower body fat mass in randomised controlled trials ${ }^{(31-33)}$, the number of studies for dairy subtypes is limited. We found no significant associations between any dairy type and total or peripheral fat mass or waist circumference and waist:hip ratio as proxies for fat mass distribution. The direction of the associations we observed between low-fat dairy consumption and total body fat mass, and waist circumference was consistent with the findings of previous studies and with our findings for VAT:SCAT. The higher dairy amounts used in feeding trials compared with the consumption levels reported in observational studies could partly explain the lack of significance in certain associations. In addition, it is expected that the direction of the association of low-fat dairy products with VAT:SCAT is consistent with that of the association of low-fat dairy products with waist circumference and the waist:hip ratio, because waist circumference is a proxy measure of abdominal fat. VAT:SCAT was estimated with higher accuracy than waist and hip circumferences. This might partly explain as to why the association with VAT:SCAT was significant after multiple test correction, but not the association with waist circumference and the waist:hip ratio.

No mechanism has been reported for an inverse association between low-fat dairy consumption and VAT:SCAT. Nevertheless, a plausible explanation could be that VAT is more metabolically active with a more efficient glucose uptake than SCAT ${ }^{(34)}$, and therefore the effects of dairy nutrients on fat mass are more pronounced in the VAT than in SCAT. Among dairy nutrients, Ca may reduce the fat content of the adipose tissue because intracellular $\mathrm{Ca}$ promotes lipolysis and reduces lipogenesis ${ }^{(35)}$ and $\mathrm{Ca}$ supplementation was previously shown to decrease VAT without changing body weight or total abdominal fat ${ }^{(36)}$.
With our analyses and specifically the observed positive association between milk consumption and body lean mass, we extend the previous understanding on the positive association between total dairy consumption and total lean mass ${ }^{(31)}$ to include specific dairy subtypes. Dairy products and mainly milk have been consistently associated with bone health due to their nutrients content including Ca, P, vitamin D and protein, which might partly explain the positive association with lean mass ${ }^{(37)}$. Another potential mechanism is the increasing effect of milk on growth hormone ${ }^{(38,39)}$, which has been associated with a higher lean mass through a higher bone mineral density and muscle $\operatorname{mass}^{(40)}$.

\section{Strengths and limitations}

Our study has several strengths including its large sample size ( $n 12$ 065), and the inclusion of several dairy subtypes, which allowed the investigation of potentially different associations with adiposity for different dairy types. By employing DEXA, we were able to use more accurate methods to assess VAT and SCAT than previous studies that used waist circumference and waist:hip ratio as proxies of central adiposity ${ }^{(12)}$. Our statistical approaches were thorough including the adjustment for important potential confounders including objectively measured physical activity, the derivation of estimates robust to outliers and the handling of missing data with multiple imputations.

Our study also has limitations. The cross-sectional design increases the risk of reverse causation and limits inference for causal association and our results can thus be used mainly for hypothesis generation. Although the FFQ used was assessed for validity in a similar population ${ }^{(18)}$ and we adjusted for BMI as an established factor of dietary misreporting ${ }^{(41)}$, we cannot exclude the possibility of error due to dietary misreporting caused by the use of self-reported methods of dietary assessment. Commensurate with this, the FFQ was limited in the detail on types of dairy products consumed, so we were not able to estimate the nutrient content accurately and thus made assumptions based on average estimates. The questionnaire also limited the assessment of various dairy definitions (e.g. sweetened yogurt) and the influences of such variations on study results. Although we adjusted for many potential confounders, residual confounding cannot be ruled out. We were also not able to disentangle whether BMI was a confounder, a mediator or both in our cross-sectional analysis, while we could examine the associations independent of BMI. If BMI is on the causal pathway, adjustment for BMI would have attenuated the findings towards the null. Future prospective analyses of change in weight (or BMI) in relation to dairy products will be better placed to investigate this further. The response rate of our study was moderate at $27 \%$. This should be placed in the context of the internal validity of our study with our use of objective assessments, that is, DEXA on the full cohort, but we acknowledge limited external validity (generalisability) of our findings for the UK general population. Moreover, our population was largely White European, who overall consume higher amounts of dairy products compared with other populations especially in South Asia, South America and Africa ${ }^{(42)}$, so generalisability of our results to other ethnic groups with different consumption patterns might be 
limited. Specifically, consumption of different dairy groups has a limited range in our study, with low levels of high-fat dairy products and high levels of low-fat dairy products. This might compromise the power to detect associations across a broader range of dairy consumption, which might be seen in other populations.

\section{Conclusion}

We observed an inverse association between low-fat dairy products and the ratio of visceral to subcutaneous fat, which suggests that abdominal obesity may be a potential pathway for the association of dairy consumption with cardiometabolic disease. In addition, the observed association of higher milk consumption with a higher body lean mass could also be a potential explanation for the overall metabolic associations observed for dairy consumption. These findings are important for hypothesis generation and should stimulate further investigation in prospective studies, clinical trials and mechanistic studies of the link between dairy products and cardiometabolic disease.

\section{Acknowledgements}

The authors thank Abigail Britten, Vasileios Kaimakis, Steven Knighton, Richard Powell, Adam Dickinson, Susie Boatman and Inge Loudon for technical assistance as members of the Fenland Study team.

The Fenland Study was funded by the Medical Research Council and the Wellcome Trust. The present work was supported by the Medical Research Council (N. G. F., grant number MC_UU_12015/5) (N. J. W., grant number MC_UU_12015/1) (S. B., grant number MC_UU_12015/3); the National Institute of Health Research Cambridge (NIHR) Biomedical Research Centre (N. G. F., S. B. and N. J. W., grant number IS-BRC1215-20014); and the Cambridge Trust (E. T.). The funders had no role in the design, analysis or writing of this article.

N. J. W., N. G. F., S. J. G. and S. B. designed research, and N. G. F., F. I. and E. T. designed the study question; E. D. L. R. provided specialist input to anthropometric measurements; E. T. and F. I. analysed data; E. T., F. I. and N. G. F. drafted the manuscript; E. T. had primary responsibility for final content. All authors read and approved the final version.

There were no conflicts of interest.

\section{Supplementary material}

For supplementary material/s referred to in this article, please visit https://doi.org/10.1017/S0007114519001776

\section{References}

1. Forouzanfar MH, Alexander L, Anderson HR, et al. (2015) Global, regional, and national comparative risk assessment of 79 behavioural, environmental and occupational, and metabolic risks or clusters of risks in 188 countries, 1990-2013: a systematic analysis for the Global Burden of Disease Study 2013. Lancet 386, 2287-2323.

2. Rice BH, Quann EE \& Miller GD (2013) Meeting and exceeding dairy recommendations: effects of dairy consumption on nutrient intakes and risk of chronic disease. Nutr Rev $\mathbf{7 1}$, 209-223.

3. Ramsden CE, Zamora D, Majchrzak-Hong S, et al. (2016) Re-evaluation of the traditional diet-heart hypothesis: analysis of recovered data from Minnesota Coronary Experiment (1968-73). BMJ 353, i1246.

4. Guo J, Astrup A, Lovegrove JA, et al. (2017) Milk and dairy consumption and risk of cardiovascular diseases and all-cause mortality: dose-response meta-analysis of prospective cohort studies. Eur J Epidemiol 32, 269-287.

5. Alexander DD, Bylsma LC, Vargas AJ, et al. (2016) Dairy consumption and CVD: a systematic review and meta-analysis. Br J Nutr 115, 737-750.

6. Aune D, Norat T, Romundstad P, et al. (2013) Dairy products and the risk of type 2 diabetes: a systematic review and dose-response meta-analysis of cohort studies. Am J Clin Nutr 98, 1066-1083.

7. Gijsbers L, Ding EL, Malik VS, et al. (2016) Consumption of dairy foods and diabetes incidence: a dose-response meta-analysis of observational studies. Am J Clin Nutr $\mathbf{1 0 3}$, 1111-1124.

8. Gao D, Ning N, Wang C, et al. (2013) Dairy products consumption and risk of type 2 diabetes: systematic review and dose-response meta-analysis. PLOS ONE 8, e73965.

9. Mozaffarian D (2016) Dietary and policy priorities for cardiovascular disease, diabetes, and obesity a comprehensive review. Circulation 133, 187-225.

10. Geng T, Qi L \& Huang T (2018) Effects of dairy products consumption on body weight and body composition among adults: an updated meta-analysis of 37 randomized control trials. Mol Nutr Food Res 62, 1700410.

11. Stonehouse W, Wycherley T, Luscombe-Marsh N, et al. (2016) Dairy intake enhances body weight and composition changes during energy restriction in 18-50-year-old adults - a metaanalysis of randomized controlled trials. Nutrients $\mathbf{8}, 394$.

12. Wajchenberg BL (2000) Subcutaneous and visceral adipose tissue: their relation to the metabolic syndrome. Endocr Rev 21, 697-738.

13. Fox CS, Massaro JM, Hoffmann U, et al. (2007) Abdominal visceral and subcutaneous adipose tissue compartments. Circulation 116, 39-48.

14. Porter SA, Massaro JM, Hoffmann U, et al. (2009) Abdominal subcutaneous adipose tissue: a protective fat depot? Diabetes Care 32, 1068-1075.

15. Kaess B, Pedley A, Massaro J, et al. (2012) The ratio of visceral to subcutaneous fat, a metric of body fat distribution, is a unique correlate of cardiometabolic risk. Diabetologia $\mathbf{5 5}$, 2622-2630.

16. Josse AR, Atkinson SA, Tarnopolsky MA, et al. (2011) Increased consumption of dairy foods and protein during diet-and exercise-induced weight loss promotes fat mass loss and lean mass gain in overweight and obese premenopausal women. J Nutr 141, 16261634.

17. Pallister T, Jackson M, Martin T, et al. (2017) Untangling the relationship between diet and visceral fat mass through blood metabolomics and gut microbiome profiling. Int J Obes $\mathbf{4 1}$, 1106.

18. Bingham SA, Welch AA, McTaggart A, et al. (2001) Nutritional methods in the European prospective investigation of cancer in Norfolk. Public Health Nutr 4, 847-858.

19. Mulligan AA, Luben RN, Bhaniani A, et al. (2014) A new tool for converting food frequency questionnaire data into nutrient and food group values: FETA research methods and availability. BMJ Open 4, e004503.

20. O'Connor LM, Lentjes MA, Luben RN, et al. (2014) Dietary dairy product intake and incident type 2 diabetes: a prospective 
study using dietary data from a 7-day food diary. Diabetologia 57, 909-917.

21. Clifton E, Day F, Rolfe EDL, et al. (2017) Associations between body mass index-related genetic variants and adult body composition: the Fenland cohort study. Int J Obes 41, 613-619.

22. Kaul S, Rothney MP, Peters DM, et al. (2012) Dual-energy X-ray absorptiometry for quantification of visceral fat. Obesity (Silver Spring) 20, 1313-1318.

23. Mohammad A, De Lucia Rolfe E, Sleigh A, et al. (2017) Validity of visceral adiposity estimates from DXA against MRI in Kuwaiti men and women. Nutr Diabetes 7, e238.

24. Brage S, Brage N, Franks P, et al. (2005) Reliability and validity of the combined heart rate and movement sensor Actiheart. Eur J Clin Nutr 59, 561-570.

25. Brage S, Ekelund U, Brage N, et al. (2007) Hierarchy of individual calibration levels for heart rate and accelerometry to measure physical activity. J Appl Physiol 103, 682-692.

26. White IR, Royston P \& Wood AM (2011) Multiple imputation using chained equations: issues and guidance for practice. Stat Med 30, 377-399.

27. Verardi V \& Croux C (2008) Robust regression in Stata. Stata J9, 439-453.

28. Benjamini Y \& Yekutieli D (2001) The control of the false discovery rate in multiple testing under dependency. Ann Stat 29, 1165-1188.

29. Willett WC, Howe GR \& Kushi LH (1997) Adjustment for total energy intake in epidemiologic studies. Am J Clin Nutr $\mathbf{6 5}$, 1220S-1228S.

30. Livingstone MB \& Black AE (2003) Markers of the validity of reported energy intake. J Nutr 133, Suppl. 3, 895S-920S.

31. Abargouei AS, Janghorbani $\mathrm{M}$, Salehi-Marzijarani $\mathrm{M}$, et al. (2012) Effect of dairy consumption on weight and body composition in adults: a systematic review and meta-analysis of randomized controlled clinical trials. Int J Obes (Lond) $\mathbf{3 6}$, $1485-1493$.

32. Booth AO, Huggins CE, Wattanapenpaiboon N, et al. (2015) Effect of increasing dietary calcium through supplements and dairy food on body weight and body composition: a meta-analysis of randomised controlled trials. Br J Nutr 114, 1013-1025.

33. Chen M, Pan A, Malik VS, et al. (2012) Effects of dairy intake on body weight and fat: a meta-analysis of randomized controlled trials. Am J Clin Nutr 96, 735-747.

34. Christen T, Sheikine Y, Rocha VZ, et al. (2010) Increased glucose uptake in visceral versus subcutaneous adipose tissue revealed by PET imaging. JACC Cardiovasc Imag 3, 843-851.

35. Zemel MB (2003) Role of dietary calcium and dairy products in modulating adiposity. Lipids 38, 139-146.

36. Rosenblum JL, Castro VM, Moore CE, et al. (2012) Calcium and vitamin $\mathrm{D}$ supplementation is associated with decreased abdominal visceral adipose tissue in overweight and obese adults. Am J Clin Nutr 95, 101-108.

37. Thorning TK, Raben A, Tholstrup T, et al. (2016) Milk and dairy products: good or bad for human health? An assessment of the totality of scientific evidence. Food Nutr Res 60, 32527.

38. Rich-Edwards JW, Ganmaa D, Pollak MN, et al. (2007) Milk consumption and the prepubertal somatotropic axis. Nutr J 6, 28.

39. Van Vught A, Nieuwenhuizen A, Veldhorst M, et al. (2010) The effects of dietary protein on the somatotropic axis: a comparison of soy, gelatin, [alpha]-lactalbumin and milk. EurJClin Nutr 64, 441.

40. Carroll PV, Christ the members of Growth Hormone Research Society Scientific Committee ER, Bengtsson BA, et al. (1998) Growth hormone deficiency in adulthood and the effects of growth hormone replacement: a review. J Clin Endocrinol Metab 83, 382-395.

41. Trijsburg L, Geelen A, Hollman PC, et al. (2016) BMI was found to be a consistent determinant related to misreporting of energy, protein and potassium intake using self-report and duplicate portion methods. Public Health Nutr 20, 1-10.

42. Global Dietary Database (2010) Global Distribution of Milk Consumption in 2010. https://www.globaldietarydatabase.org/our-data/data-visualizations/dietary-data-country (accessed June 2018). 